\title{
Axonal Trajectories and Distribution of GABAergic Spinal Neurons in Wildtype and Mutant Zebrafish Lacking Floor Plate Cells
}

\author{
ROBERT R. BERNHARDT, CHETAN K. PATEL, STEPHEN W. WILSON, \\ AND JOHN Y. KUWADA \\ Department of Biology, The University of Michigan, Ann Arbor, Michigan 48109-1048 \\ (R.R.B., C.K.P., J.Y.K.); Neurobiology, ETH-Hönggerberg, HPM, CH-8093 Zürich, \\ Switzerland (R.R.B.); Development Biology Research Center, Division of Biomedical Sciences, \\ Kings College, London, WC2B United Kingdom (S.W.W.)
}

\begin{abstract}
The role of the midline floor plate cells in the neuronal differentiation of the spinal cord was examined by comparing putative GABAergic neurons in wildtype zebrafish embryos with those in $c y c-1$ mutant embryos. The mutation produces a pleiotropic recessive lethal phenotype and is severe in rostral brain regions, but its direct effect in the caudal hindbrain and the spinal cord is apparently restricted to the depletion of the midline floor plate cells. In wildtype embryos, an antibody against the neurotransmitter GABA labeled the cell bodies, axons, and growth cones of three classes of previously identified neurons; dorsal longitudinal neurons (DoLA), commissural secondary ascending neurons (CoSA), and ventral longitudinal neurons (VeLD). A novel ventral cell type, Kolmer-Agduhr (KA) neurons, was also labeled. In the cyc-1 mutant, abnormalities were observed in some, but not all, of the GABAreactive CoSA, VeLD, and KA axons, while the axonal trajectories of DoLA neurons were not affected. Furthermore, the number of KA cells was reduced in the mutant while the numbers of the other GABAreactive cells were unperturbed. These observations corroborate our earlier hypothesis that the floor plate cells are one of several guidance cues that direct axonal outgrowth near the ventral midline of the spinal cord. They also suggest that the floor plate cells may play a role in the cellular differentiation of the spinal cord of zebrafish embryos. @ 1992 Wiley-Liss, Inc.
\end{abstract}

Key words: axonal outgrowth, neuronal differentiation, zebrafish, growth cones, spinal cord

During the development of the nervous system an undifferentiated neuroepithelium gives rise to specific neuronal cell types, which then establish specific connections. One approach to identifying the mechanisms which determine neuronal identity and connectivity is to examine a system whose cellular anatomy is known in great detail. Ideally, such a system also should be amenable to manipulation in order to test the importance of specific interactions between any given developing neuron and its environment. These interactions may be responsible for determining neuronal fate as well as the precise patterns of axonal outgrowth. This approach has been successfully applied to the study of invertebrate neuronal development (see Goodman et al., '84), as well as to the analysis of some embryonic vertebrate nervous systems (Eisen, '91; Chitnis and Kuwada, '91).

In the early spinal cord of zebrafish embryos, a small number of identified neurons account for the majority of the postmitotic cells present (Bernhardt et al., '90). De- tailed cellular analysis of the embryonic zebrafish spinal cord has served as the basis for experimental studies of the cues that direct axonal outgrowth. One focus of these studies has been the interactions of the spinal neurons CoPA and VeLD with the floor plate cells which occupy the ventral midline of the spinal cord. The floor plate cells are of interest because they are the site of cell-specific turns by CoPA and VeLD growth cones (Kuwada et al., '90). Following elimination of floor plate cells either by laser ablations or by the cyc-1 (b16) mutation (Hatta et al., '91), the VeLD and CoPA growth cones dramatically increased the number of errors at the ventral midline (Bernhardt et al., '92). This and analogous experiments following elimination of floor plate cells in the zebrafish hindbrain (Hatta et al., '90)

Accepted August 5, 1992.

Address correspondence to Robert R. Bernhardt, Neurobiology, ETHHönggerberg, HPM, CH-8093 Zürich, Switzerland. 
demonstrated that floor plate cells were an important source of guidance cues for these growth cones. Similarly the floor plate, possibly together with the notochord, is involved in guiding axonal outgrowth in a several other vertebrates (Xenopus: Clarke et al., '91; chick: TessierLavigne et al., '88; Placzek et al., '90; Yamada et al., '91; Yaginuma and Oppenheim, '91, mouse: Bovalenta and Dodd, '91; and rat: Bovalenta and Dodd, '90). However, since a significant number of VeLD and CoPA growth cones behaved normally at the ventral midline despite the floor plate deficit, it is possible that another source of guidance cues at the ventral midline may also be capable of guiding these growth cones (Bernhardt et al., '92). Redundant sources of guidance cues may also direct growth cones in the zebrafish brain (Chitnis and Kuwada, '91).

In addition to its role in axonal guidance, the floor plate, possibly together with the notochord, has been shown to regulate the differentiation of cells in the ventral spinal cord in several vertebrate embryos (chick: van Straaten et al., '89; Hirano et al., '91; Yamada et al., '91; Xenopus: Clarke et al., '91; rat: Bovalenta and Dodd, '91). For example, in the chick, an elegant series of experiments demonstrated that the floor plate induces spinal motor neurons (Yamada et al., '91). Following elimination of the floor plate, motor neurons failed to differentiate. However, primary motor neurons in the zebrafish spinal cord are not affected by the removal of floor plate cells (Eisen, '91; Bernhardt et al., '92). This may be due to the early birth date and development of these motor neurons. Whether the floor plate may induce later differentiating neurons in the ventral cord of the zebrafish is unknown.

Because changes in the environment may affect neuronal morphology, cell specific markers are important in identifying neurons and their axons in situations where the normal cellular environment has been altered. A monoclonal antibody, CON1, which labels a subset of early spinal neurons and their axons (Bernhardt et al., '90; Rosenblatt et al., submitted), has been a valuable tool in studies of axonal outgrowth in mutant and experimental spinal cords (Bernhardt et al., '92). Antibodies against a number of neurotransmitters have been useful in identifying classes of neurons and in characterizing their axonal outgrowth in embryonic Xenopus spinal cord (Gallagher and Moody, '87; Roberts et al., '87; Roberts et al., '88). Here we report that GABA labeling identifies four classes of spinal neurons in the zebrafish embryo, two of which are apparent homologues of GABA reactive neurons described previously in Xenopus spinal cord (Dale et al., '87a,b; Roberts et al., '88).

In order to examine further the role of the floor plate for the development of the zebrafish spinal cord we have

Abbreviations

$\begin{array}{ll}\text { CoPA } & \text { commissural primary ascending neuron } \\ \text { CoSA } & \text { commissural secondary ascending neuron } \\ \text { DoLA } & \text { dorsal longitudinal neuron } \\ \text { KA } & \text { Kolmer-Agduhr neuron } \\ \text { RB } & \text { Rohon-Beard neuron } \\ \text { VeLD } & \text { ventral longitudinal neuron }\end{array}$

Cell types, which were first described in zebrafish, are named according to the soma position (dorsal or ventral), the time of axonogenesis (primary are early, secondary are later), and axonal trajectories (commissural or longitudinal). Commissural cells have dorsal cell bodies. For cell types which had been identified previously in fish (Rohon-Beard neurons) or which have homologues in amphibians (Kolmer-Agduhr neurons), the established nomenclature is used. followed the development of GABAergic neurons, both in the presence and absence of floor plate cells. First, we wanted to know whether the growth cones of classes of spinal neurons other than CoPA and VeLD also relied on the floor plate for guidance. Second, we wanted to test whether the floor plate may have a role in the induction and maturation of later differentiating neurons. GABA labeling in wildtype and $c y c-1$ embryos suggested that the floor plate guides the growth cones of two other spinal neurons, CoSA and KA, and that floor plate cells are necessary for the appearance of the full complement of KA cells.

\section{MATERIALS AND METHODS Animals}

Zebrafish embryos were collected from a laboratory breeding colony, raised at $28.5^{\circ} \mathrm{C}$, and staged from the estimated time of fertilization (Myers et al., '86). Homozygotic cyc-1 (b16) mutant embryos were obtained by mating heterozygotic parents and identified by their cyclopean eye phenotype (Hatta et al., '91).

\section{Immunohistochemistry}

Embryos were labeled with a commercially available polyclonal antibody against GABA raised in guinea pigs (Eugene Tech). A mouse monoclonal antibody (MAb) against acetylated $\alpha$-tubulin (Piperno and Fuller, '85) and MAb CON1 (Rosenblatt et al., submitted) were used in combination with the GABA antibody for double labeling studies. Embryos were labeled as wholemounts, following the procedure described by Patel and co-authors (Patel et al., '89). The cells labeled by the GABA antibody were visualized with a diaminobenzidene (DAB) reaction product following application of a horseradish peroxidase conjugated antiguinea pig IgG. The mouse MAbs were visualized by using a TRITC-conjugated secondary antibody. For double staining the polyclonal GABA antibody and one of the MAbs were applied simultaneously, as were the respective secondary antibodies (HRP conjugated anti-guinea pig and TRITC conjugated anti-mouse). The $\mathrm{DAB}$ reaction did not noticeably diminish the TRITC fluorescence. Double labeled preparations were examined by switching from transmittent light to epifluorescent illumination. For sectioning, GABA labeled wholemounts were postfixed in phosphate buffered $1 \%$ glutaraldehyde/4\% paraformaldehyde, osmicated for $1 \mathrm{~h}$ ( $1 \%$ OsO4 in phosphate buffer), and embedded in Epon. Semithin sections were cut at $2 \mu \mathrm{m}$.

\section{Numerical analysis of neurons and axons}

Immunoreactive neurons were counted under the microscope by focussing through wholemounted embryos labeled with either the GABA or tubulin antibodies. Neuronal cell types could be identified unambiguously by the positions and shapes of their somata and by their characteristic axonal trajectories (see Bernhardt et al., '90). Counts were compared by using Student's t-test.

CoSA, DoLA, and VeLD neurons were counted only on one side of the spinal cord, yielding numbers per hemisegment. 'The comparison, in the wildtype cord, of the number of GABA labeled commissural neurons to the total of tubulin labeled ccmmissural neurons (Table 1) is based on counts in hemisegments $7-10$ of $27 \mathrm{~h}$ embryos. To compare the numbers of neurons in wildtype and in cyc-1 spinal cords (Table 2), all GABA labeled CoSA and DoLA neurons were counted in the hemisegments 6-10 of wholemounted 
TABLE 1. Only a Subset of All Commissural Cells Are Labeled by the GABA Antibody ${ }^{1}$

\begin{tabular}{llcrl}
\hline Cell type & Antibody & $\begin{array}{c}\text { Hemisegments } \\
\text { scored }\end{array}$ & Mean + S.D. & $\begin{array}{c}\mathrm{n} \\
\text { animals }\end{array}$ \\
\hline Commissural cells & Anti-tubulin & $7-10$ & $21.5 \pm 2.08$ & 4 \\
Commissural cells & Anti-GABA & $7-10$ & $7.7 \pm 2.12$ & 9 \\
\hline
\end{tabular}

${ }^{1}$ The comparison of the numbers of GABA- and tubulin-labeled commissural neurons in the midtrunk region of wildtype embryos reveals significantly different densities $(\mathrm{df}=11$, $\mathrm{t}=10.02, P \leq 0.001 ;$ for details of the scoring see Materials and Methods).

TABLE 2. The Density of KA Neurons is Reduced in cyc- $I$ Embryos; the Densities of Other Cell Types Are Normal ${ }^{1}$

\begin{tabular}{lclr}
\hline Cell type & $\mathrm{n}$ animals & Segments scored & Mean + S.D. \\
\hline CoSA & 9 wildtype & Hemisegments 6-10 & $9.44 \pm 2.24$ \\
& 11 cyc-l & Hemisegments 6-10 & $9.55 \pm 1.90$ \\
DoLA & 9 wildtype & Hemisegments 6-10 & $3.89 \pm 1.17$ \\
& 11 cyc-1 & Hemisegments 6-10 & $4.82 \pm 1.78$ \\
VeLD & 18 wildtype & Hemisegments 8-14 & $2.44 \pm 1.39$ \\
& 18 cyc-1 & Hemisegments 8-14 & $2.61 \pm 0.86$ \\
KA & 12 wildtype & Segments 6-10 & $38.58 \pm 7.33$ \\
& 15 cyc-1 & Segments 6-10 & $24.07 \pm 6.92$ \\
\hline
\end{tabular}

'The comparison of the total numbers of GABA labeled neurons in the trunk regions of wildtype and $c y c-1$ embryos reveals a significant difference in the number of KA neurons $(\mathrm{df}=25, \mathrm{t}=5.07, P \leq 0.001)$. The numbers for $\operatorname{CoSAs}(\mathrm{df}=18, \mathrm{t}=0.06, P \geq 0.3$ ) DoLAs $(\mathrm{df}=18, \mathrm{t}=1.28, P \geq 0.01$ ), and VeLDs ( $\mathrm{df}=34, \mathrm{t}=0.45, P \geq 0.3)$ are not significantly different (for details of the scoring see Materials and Methods).

TABLE 3. Abnormal CoSA Trajectories Are Frequent in $c y c-1$ Spinal Cords ${ }^{1}$

\begin{tabular}{|c|c|c|c|c|c|c|}
\hline & \multicolumn{2}{|c|}{ Contralateral } & \multicolumn{2}{|c|}{ Ipsilateral } & \multirow{2}{*}{$\begin{array}{l}\text { Longi- } \\
\text { tudinal }\end{array}$} & \multirow[b]{2}{*}{ Other } \\
\hline & Ascending & Descending & Ascending & Descending & & \\
\hline Wildtys & $94 \%$ (41) & - & $2 \%(1)$ & $2 \%(1)$ & - & $2 \%(1)$ \\
\hline$c y c-1$ & $42 \%(12)$ & $14 \%(4)$ & $14 \%(4)$ & $10 \%(3)$ & $17 \%(5)$ & $3 \%(1)$ \\
\hline
\end{tabular}

${ }^{1}$ GABA-labeled CoSA axons were scored in 19 wildtype and in 10 cyc-1 embryos; for details for the axonal trajectories see text.

$27 \mathrm{~h}$ wildtype and $c y c-1$ mutant embryos. VeLD neurons were most readily identified in younger embryos. Accordingly, the total number of labeled VeLDs was determined in hemisegments 8-14 of 19-22 h wildtype and cyc-1 mutant embryos. The very ventrally located KA cells were counted on both sides of the spinal midline to avoid possible ambiguities in the attribution of laterality in the cyc-1 mutant cord, where the definitive midline marker, the midline floor plate cells, is absent. Accordingly, Table 2 lists the total number of labeled KA neurons in the midtrunk segments 6-10.

The axonal trajectories of GABA labeled CoSA neurons (Table 3) were analyzed in wholemount preparations of 19-22 h wildtype and cyc-1 embryos. All axons which could be traced beyond the ventral midline were scored. The axonal trajectories of the KA neurons were analyzed in wholemounts of $20-22 \mathrm{~h}$ wildtype $(\mathrm{n}=15)$ and cyc- $1 \mathrm{em}$ bryos $(n=8)$. All axons whose directionally (ascending or descending) could be determined were scored.

\section{RESULTS \\ The GABA antibody labels four classes of early neurons in the wildtype spinal cord}

The pattern of GABA immunoreactivity was examined in wholemount preparations of zebrafish embryos $(\mathbf{n}=143)$, fixed at $16-27 \mathrm{~h}$ post fertilization (PF). Reliable staining was first detected at 18-19 h. The cell bodies, axons, and

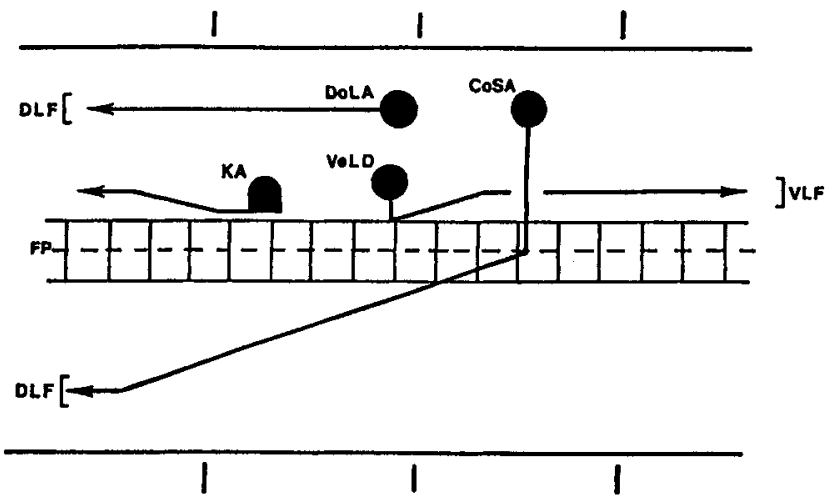

Fig. 1. Schematic diagram illustrating the GABA-reactive neurons in a $22 \mathrm{~h}$ zebrafish embryo shown in a "open-book" configuration. In this configuration the spinal cord is shown as if it were cut longitudinally along the dorsal midline, opened up, and flattened. The GABA antibody labels four types of neurons: a subclass of the commissural secondary ascending (CoSA), dorsal longitudinal ascending (DoLA), ventral longitudinal descending (VeLD), and Kolmer-Agduhr (KA) neurons. GABAreactive axons are found in two early longitudinal tracts: the dorsal (DLF) and ventral (VLF) longitudinal fasciculus. The midline floor plate cells (FP), the segment borders (bars), and the ventral midline (dashed line) are also indicated.

growth cones of developing neurons were labeled. Strong cell body labeling persisted in the $24-27 \mathrm{~h}$ cords, but axonal staining was reduced, possibly as the result of dilution due to axonal elongation. On the basis of soma position and axonal morphology (see Bernhardt et al., '90), and by comparison with neurons in the embryonic Xenopus spinal cord (Roberts et al., '87), four classes of labeled neurons were identified: VeLD, DoLA, KA, and a subset of CoSA neurons.

\section{CoSA neurons}

The zebrafish spinal cord contains two classes of early commissural neurons (Bernhardt et al., '90; Kuwada et al., '90). The large CoPA neurons project growth cones very early in development, possess a long ascending axon, and are labeled by monoclonal antibody (MAb) CON1. The smaller CoSA neurons extend their shorter axons at slightly later times and over a longer time period than CoPA cells. Furthermore, CoSAs do not label with MAb CON1 at least up to $27 \mathrm{~h} \mathrm{PF}$.

GABA labeling was first detected at $18-19 \mathrm{~h}$ in commissural neurons located in the dorsal spinal cord. The commissural axons emerged at the ventral pole of the cell body and extended ventrally (Figs. 1, 2A). Labeled commissural growth cones were often seen at the ventral midline at this time (Fig. 2B), just dorsal to the notochord and ventral to the single row of midline floor plate cells. At slightly later stages these growth cones had crossed the ventral midline and turned anteriorly (Fig. 2C). Based on soma size and the timing of axonogenesis the GABA positive commissural cells appeared to be CoSA neurons. In order to determine the identity of these cells more definitively, $27 \mathrm{~h}$ embryos were double labeled with both the GABA and the CON1 antibodies. GABA labeled commissural cells did not label with the CON1 antibody (Fig. 3A,B), demonstrating that they are indeed CoSA and not CoPA neurons.

Spinal cords were also double labeled with anti-GABA and an antibody against acetylated $\alpha$-tubulin (Piperno and Fuller, '85). This revealed that some, but not all commis- 

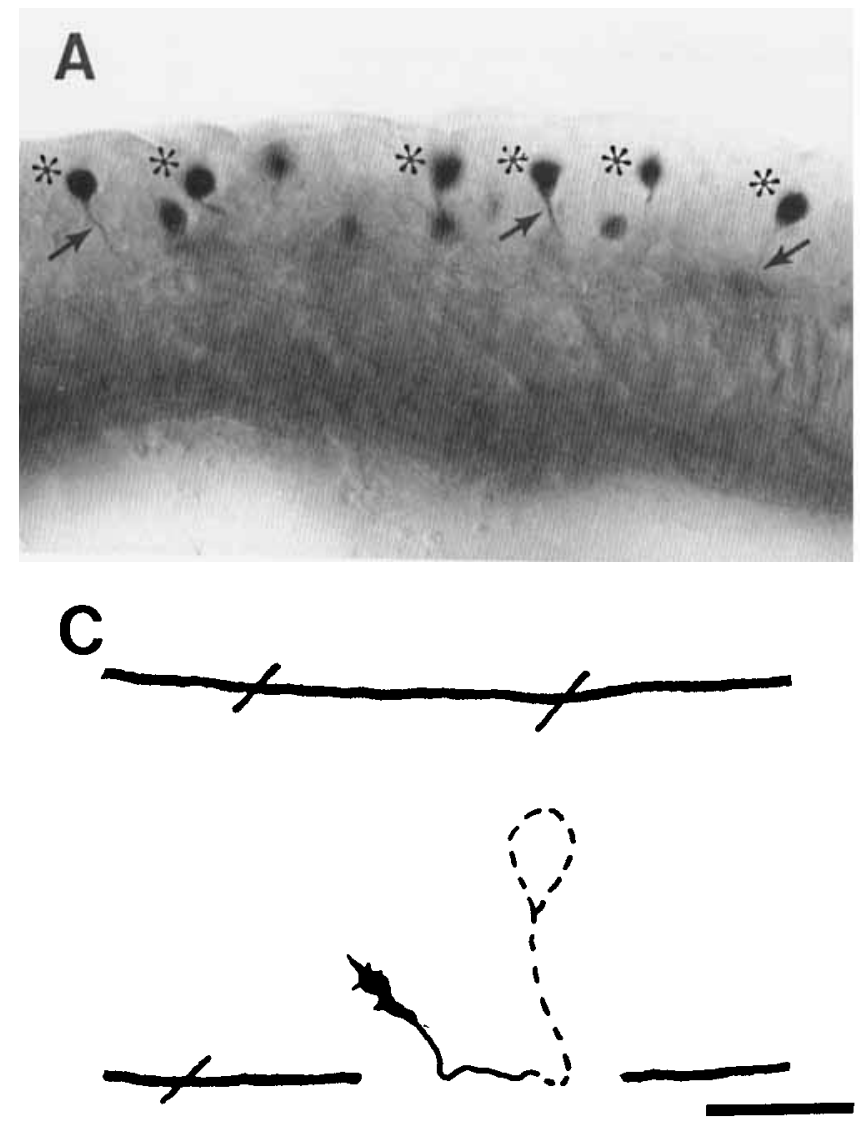

sural neurons were GABAreactive. A comparison of the complement of commissural cells labeled by either antitubulin or the GABA antibody at $27 \mathrm{~h}$ (Table 1 ) confirmed that only a subpopulation (approximately $35 \%$ ) of all commissural cells display GABA immunoreactivity. Because CoPA neurons are rare and account for only approximately $20 \%$ of commissural cells at this time (no more than one per hemisegment, Bernhardt et al., '90), this indicates that the GABA antibody labels only a subset of all CoSA neurons.

\section{VeLD neurons}

At 18-19 h GABA labeling also appeared in neurons located in the ventrolateral cord (Figs. 1, 4A; see also 2B). These neurons had relatively large, oval-shaped cell bodies. Their axons emerged at the ventral pole, initially extended ventrally towards the floor plate, and then turned caudally without crossing the ventral midline. The descending axons extended in the ventrolateral cord, forming a loosely fasciculated tract, and ended in labeled growth cones. Based on soma size, soma position, and axonal trajectory, these cells are identified as VeLD neurons (Bernhardt et al., '90).

\section{DoLA neurons}

A second class of dorsal neurons located slightly dorsal to the CoSA neurons were labeled with the GABA antibody at 20-22 $\mathrm{h}$. These dorsal cells had a long ascending axon (Figs. $1,4 \mathrm{~B}$ ), originating from the rostral pole of the soma, that extended in the dorsal longitudinal fascicle (DLF). Occasionally these axons could be traced over five or more segments. The growth cones of cells located in more rostral spinal

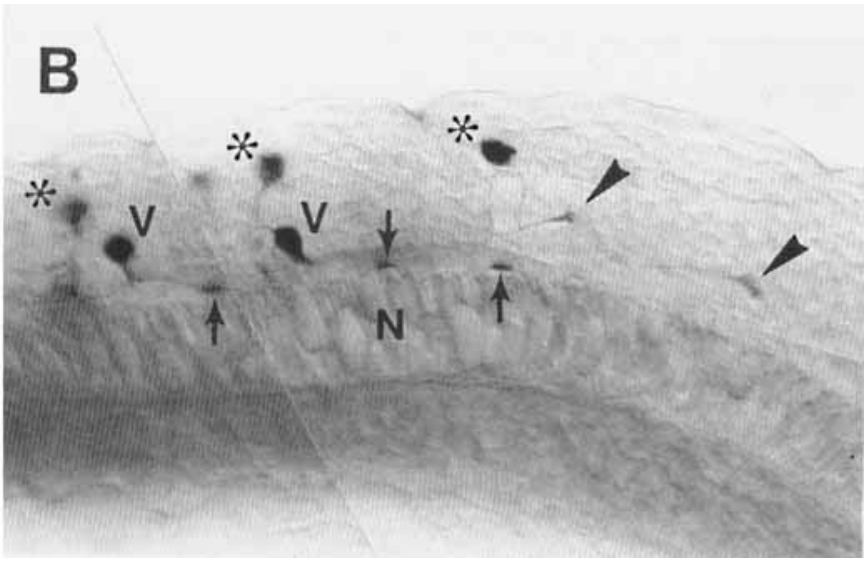

Fig. 2. The GABA antibody labels CoSA neurons and their growth cones in the developing spinal cord of zebrafish embryos. Side views of embryonic wholemounts, anterior is to the left. A: Strong labeling is detected at $19 \mathrm{~h}$ in CoSA neurons (asterisks) and their ventrally directed axons (arrows). B: At $19 \mathrm{~h}$, labeled CoSA growth cones (arrows), originating from contralateral somata, have extended to the ventral midline, just dorsal to the notochord $(\mathrm{N})$. Two VeLD neurons (V) and their descending growth cones (arrowheads) are also labeled, as are ipsilateral CoSAs (asterisks). C: Camera lucida tracing of a $20 \mathrm{~h}$ spinal cord. A labeled growth cone, originating from a contralateral cell body (broken lines) has crossed the ventral midline and turned anterior. Bars indicate segment borders. Magnification bar for A, B, $50 \mu \mathrm{m}$; for C, $100 \mu \mathrm{m}$.

segments often reached into the hindbrain. These cells appeared to correspond to the previously described DoLA neurons (Bernhardt et al., '90).

In order to definitively differentiate the putative DoLA neurons from $\mathrm{RB}$ cells which also extend axons in the ipsilateral DLF, we examined spinal cords that were double labeled with the GABA and tubulin antibodies (Fig. 5A,B). Tubulin labeling of axons clearly revealed the morphology of the RB cells which differ form DoLAs in having a descending and a peripheral axon, as well as an ascending axon. RB neurons were not GABA immunoreactive. A cell type in the Xenopus spinal cord morphologically similar to the DoLA cells also is GABA immunoreactive (Roberts et al., '87).

\section{KA neurons}

At 20-22 h, GABA labeling was apparent in a population of cells located near the ventral midline. In sideviews of wholemounted preparations the somata appeared cuboidal and extended to the central canal (Figs. 1, 6A). Axons originated from the rostral side of the soma and ascended ipsilaterally in the ventral longitudinal fasciculus (VLF) of the ventrolateral spinal cord. By focussing through the wholemounts, it was apparent that some of the cells were located just lateral to the midline floor plate cells, while others were slightly more lateral. Examination of cross sections confirmed that some of the labeled neurons were immediately adjacent to the midline floor plate cells (Fig. $6 \mathrm{~B}$ ), interspersed in between unlabeled neuroepithelial cells previously identified as lateral floor plate cells (Kuwada et 

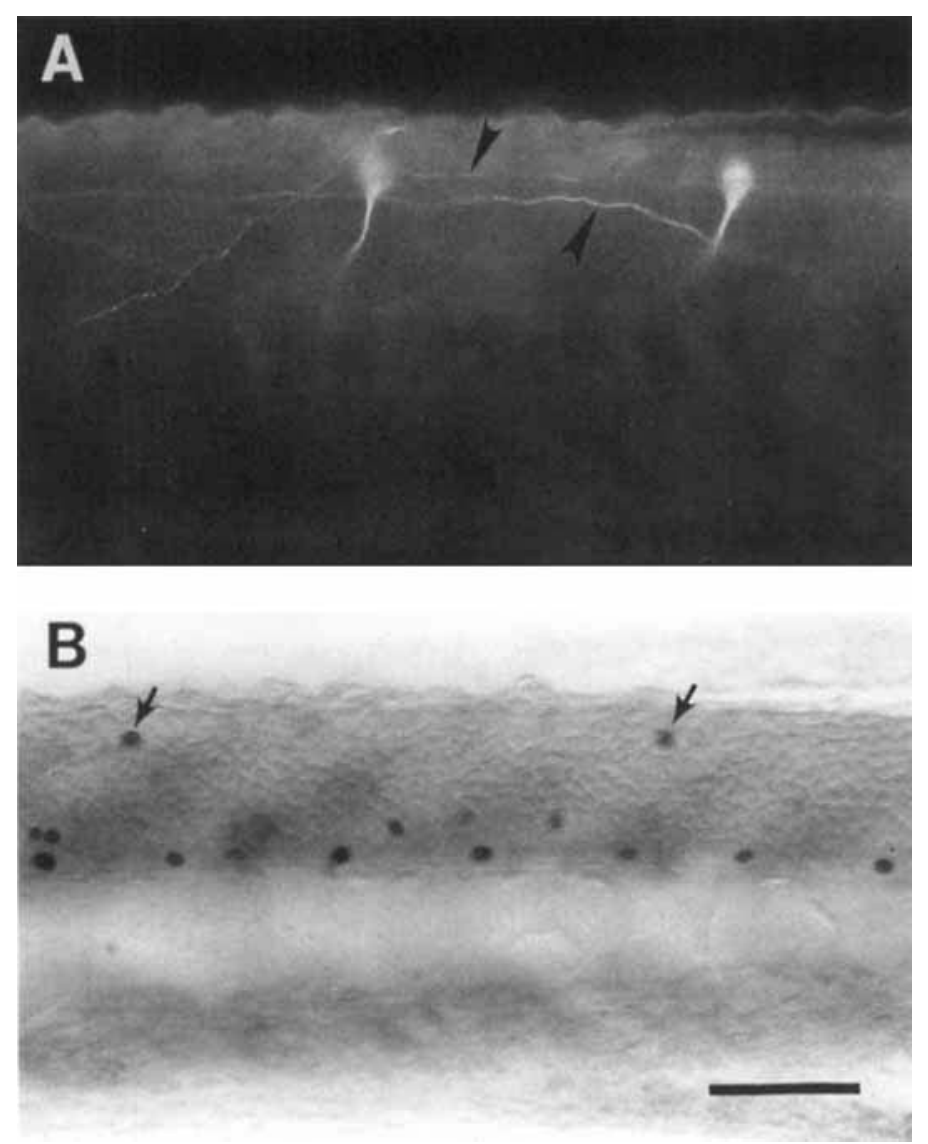

Fig. 3. Double staining, using the CON1 and GABA antibodies, demonstrates that CoPA neurons do not show GABA-immunoreactivity. Side view of the spinal cord of a wholemounted $27 \mathrm{~h}$ embryo, anterior is to the left. A: Rhodamine fluorescence reveals 2 CON1 labeled CoPA neurons and $2 \mathrm{CoPA}$ axons (arrowheads) originating from

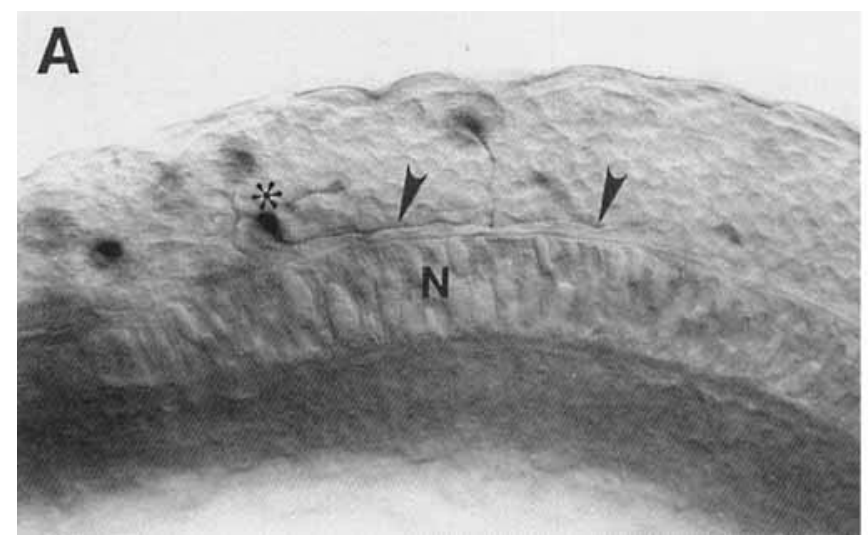

Fig. 4. The GABA antibody labels VeLD and DoLA neurons. Anterior is to the left. A: A VeLD axon originates from the ventral pole of the parent cell body (asterisks) and descends ventrolaterally (arrow-

al., '90; Bernhardt et al., '92; see also Discussion). Others were separated from the midline floor plate cell by GABAnegative cells (Fig. 6C). The cross sections also showed that the cells contacted the lumen of the central canal. These contralateral cell bodies. B: Same field and focus: GABA-immunoreactivity, revealed by $D A B$ reaction product, is detected in two dorsal neurons (arrows), but these do not correspond to the CoPAs labeled in A. KA neurons in the ventral cord are also labeled. Magnification bar, $50 \mu \mathrm{m}$.

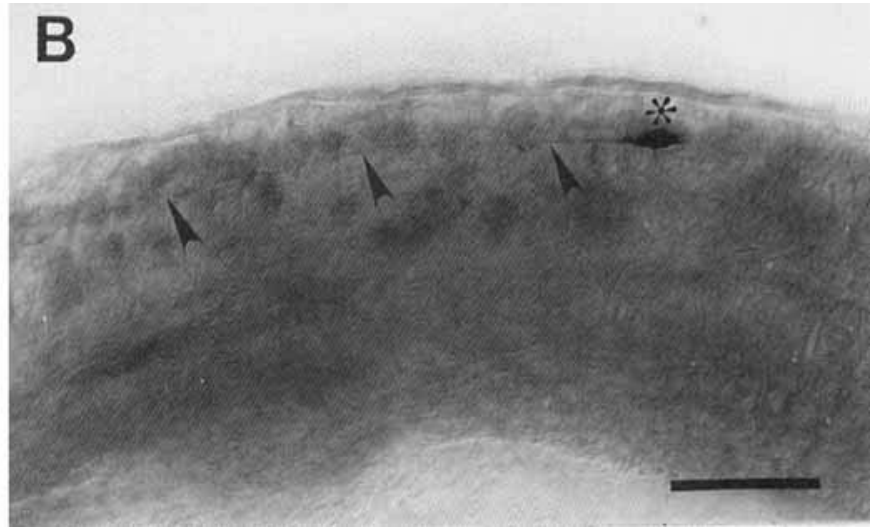

heads) in this $19 \mathrm{~h}$ cord. $\mathrm{N}$, notochord. B: A DoLA neuron (asterisks) and its long ascending axon (arrowheads) are labeled in this $22 \mathrm{~h}$ spinal cord. Magnification bar, $50 \mu \mathrm{m}$.

cells are similar to the GABAreactive KA cells found in the embryonic Xenopus spinal cord (Dale et al., '87a,b; Roberts et al., '87), and consequently have been identified as KA cells in the zebrafish spinal cord. 

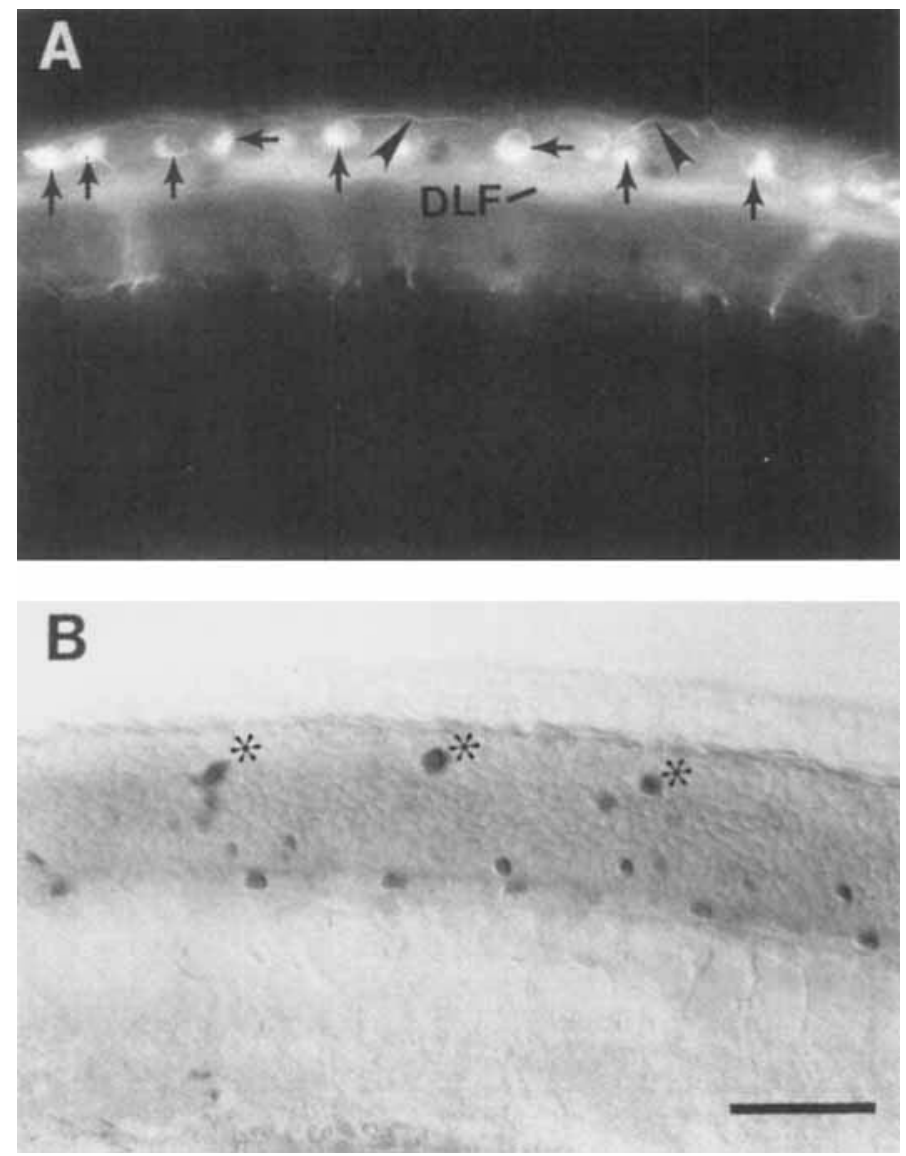

Fig. 5. Double labeling, using the tubulin and GABA antibodies, reveals that $\mathrm{RB}$ cells are not GABA-immunoreactive. Side view of the spinal cord of a wholemounted $27 \mathrm{~h}$ embryo, anterior is to the left. A Rhodamine fluorescence reveals tubulin-labeled $\mathrm{RB}$ cell bodies (arrows), their central axons (DLF), and the proximal portions of their peripheral axons (arrowheads). B: Same frame and focus: three DoLA neurons (asterisks), but none of the RB neurons, are labeled by the GABA antibody, which is visualized by $\mathrm{DAB}$ reaction product. The smaller cells in the ventral cord are KA neurons. Magnification bar, 50 $\mu \mathrm{m}$.

\section{The $c y c-1(b 16)$ mutation affects some, but not all, GABA-immunoreactive spinal neurons}

The $c y c-1$ mutation of zebrafish results in the deletion of a morphologically and immunologically distinct midline floor plate cell along the entire rostrocaudal extent of the spinal cord (Hatta et al., '91). In order to examine the effects of the mutation on GABA-immunoreactive neurons, wholemount preparations of cyc- 1 embryos $(n=52)$ at $18-27 \mathrm{~h}$ labeled with the GABA antibody were analyzed. All four classes of neurons described in the wildtype spinal cord could also be identified in the mutant spinal cord (Fig. 7A-D), but only DoLA, CoSA, and KA axons were labeled reliably enough to allow for a detailed analysis of their trajectories. Soma staining was sufficient, however, to allow for the determination of the distribution of all four cell types.

\section{DoLA and VeLD neurons}

GABA labeling revealed that the axonal trajectories and the density (Table 2) of DoLA neurons were normal. VeLD axons had previously been found to sometimes have aberrant trajectories in the mutant spinal cord (Bernhardt et al., '92). Occasionally labeling was sufficient to reveal VeLD axons with aberrant trajectories (Fig. 8C). As with DoLA neurons, the number of VeLD neurons was not affected by the mutation (Table 2).

\section{CoSA neurons}

GABA-labeled CoSA neurons were present in normal numbers in the mutant spinal cord (Table 2), but their axons often followed aberrant trajectories (defined as failing to extend directly to the ventral midline, failing to cross the ventral midline, or failing to extend rostrally). In wildtype spinal cords $6 \%$ of all GABA-labeled CoSA axons have abnormal trajectories. In cyc-1 embryos $58 \%$ of all GABA-labeled CoSA axons that could be unambiguously traced had abnormal trajectories, while the rest were normal (Table 3 ). All axons eventually reached the ventral midline region but some were abnormal in that they first extended longitudinally, either anteriorly or posteriorly, before turning ventrally (Fig. $8 \mathrm{~A}$ ). At the ventral midline, some of the GABA-labeled CoSA axons failed to cross but nevertheless turned anterior to ascend on the ipsilateral side of the spinal cord (Fig. 8B). Other axons turned posterior to descend either ipsilaterally or contralaterally (Fig. 8A), in the spinal cord. In one case a GABA-labeled CoSA neuron projected two axons ventrally (the axons 


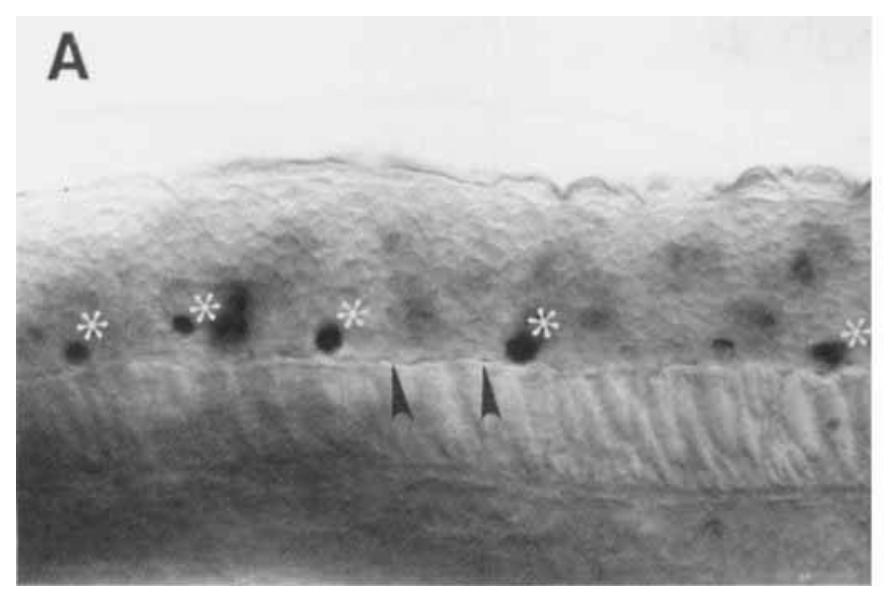

Fig. 6. KA neurons are labeled by the GABA antibody in a side view and two cross sections of embryonic spinal cords. A: KA neurons (asterisks), located near the ventral midline of a $22 \mathrm{~h}$ embryo, have ipsilaterally ascending axons (arrowheads), rostral is to the left. B: A labeled KA cell (arrow) and an unlabeled lateral floor plate cell (arrowhead) flank the midline floor plate cell (asterisk) in a $22 \mathrm{~h}$ embryo. The KA cell contacts the central canal. Toluidine blue counter-
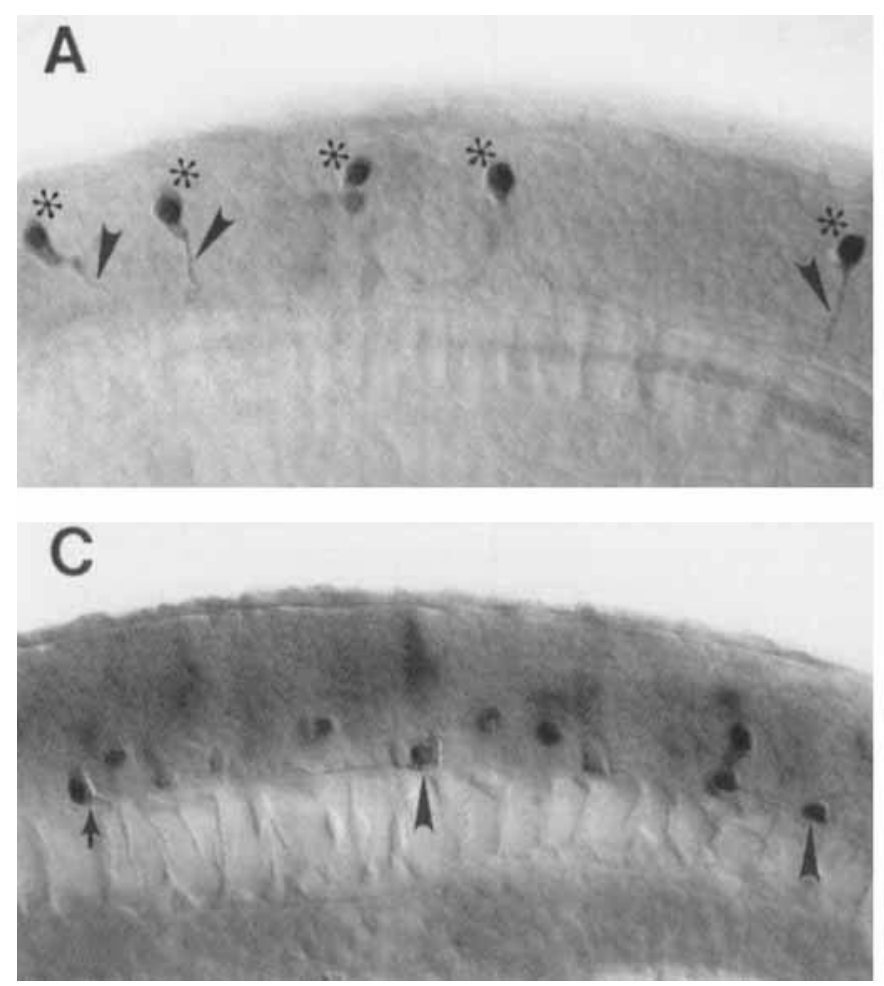

Fig. 7. The four classes of GABA-immunoreactive neurons that are found in the wildtype embryonic spinal cord can also be identified in the cyc-1 mutant. Sideviews of the spinal cords of wholemounted embryos, anterior is to the left. A: CoSA neurons (asterisks) in a $19 \mathrm{~h}$ embryo, some of the axons (arrowheads) can be traced to the ventral midline. B: VeLD neurons (asterisks) in a $19 \mathrm{~h}$ embryo, one with a ventrally emerging axon (arrowhead). Labeled neurons in the dorsal cord are CoSAs. C:

could not be traced beyond the ventral midline). The error rate of axonal outgrowth by GABAergic CoSA neurons as well as the range of abnormalities are comparable to that of CoPA axons in cyc-1 spinal cords (Bernhardt et al., '92).
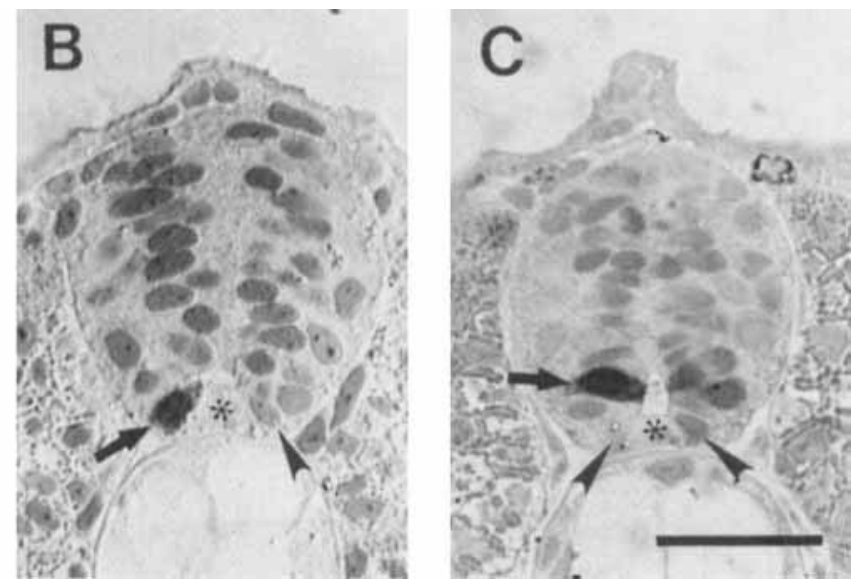

stain lightly labels nuclei, GABA immunostaining extends through the cytoplasm. C: An example, in a $27 \mathrm{~h}$ embryo, of a more dorsal KA cell (arrow), which is not apposed to the midline floor plate cell (asterisk). The cell contacts the lumen of the central canal. The two lateral floor plate cells are indicated by arrowheads. Toluidine blue counterstain. Magnification bar, $50 \mu \mathrm{m}$ for A; $25 \mu \mathrm{m}$ for B, C.
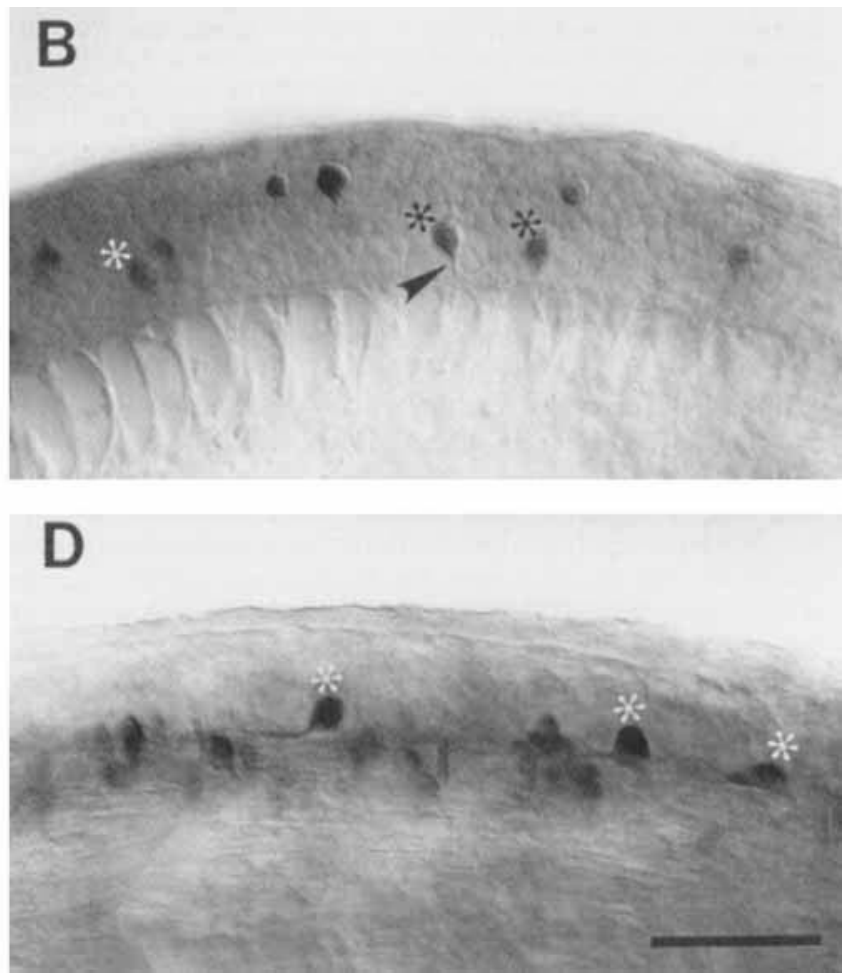

Several KA neurons are located near the ventral midline of a $22 \mathrm{~h}$ spinal cord. Ascending axons emerge laterally from 2 parent somata (arrowheads). One KA neuron gives rise to an abnormal, descending axon (arrow). N, notochord. D: DoLA neurons and their ascending axons in a $22 \mathrm{~h}$ cord are normal. Labeled CoSAs are slightly out of focus. Magnification bar, $50 \mu \mathrm{m}$.

\section{KA neurons}

KA cells could be identified in mutant spinal cords on the basis of cell body position, soma shape, and because they 


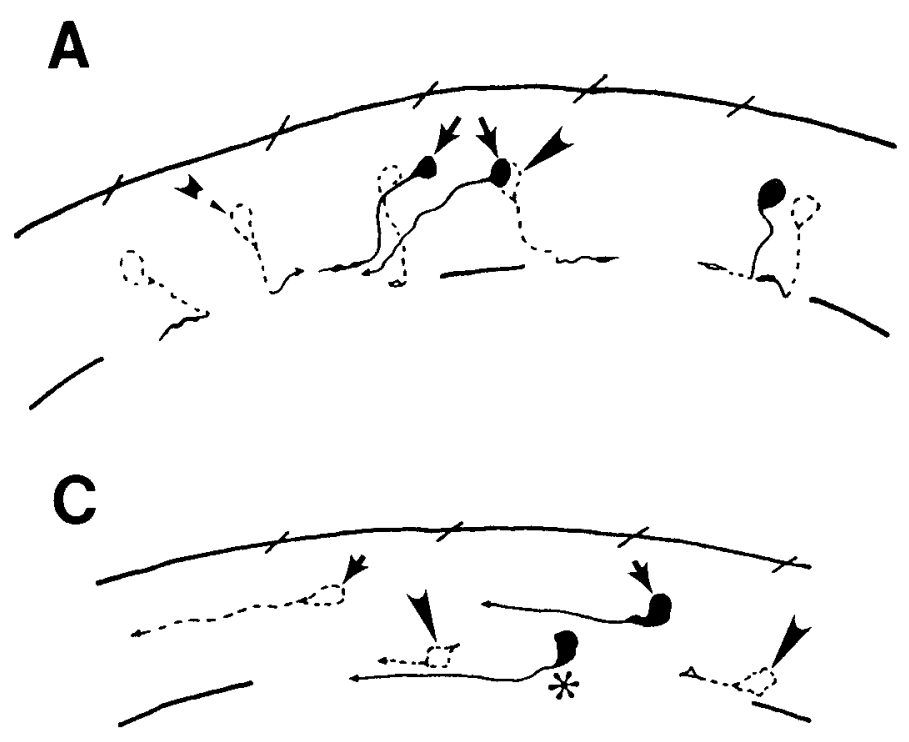

B

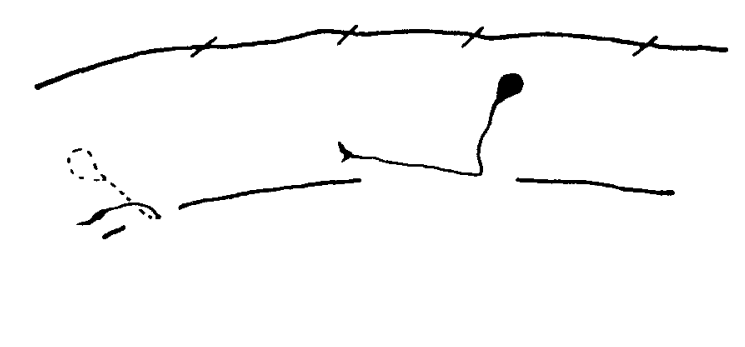

D

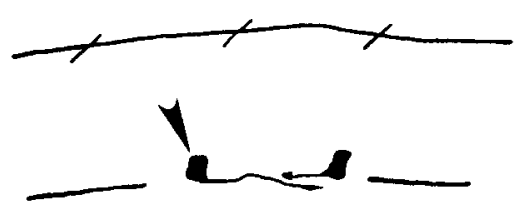

Fig. 8. CoSA, VeLD, and KA axons, but not DoLA axons, sometimes establish abnormal trajectories in cyc-1 mutant spinal cords. Camera lucida tracings of embryonic wholemounts labeled with the GABA antibody, anterior is to the left. Broken lines indicate contralateral cells or axons. A: Of 8 labeled CoSAs in this $20 \mathrm{~h}$ spinal cord, four have normal axons which have crossed the ventral midline and then turned anterior. The other four neurons are abnormal; two have axons which initially extend longitudinally (arrows), two have contralaterally descending axons (arrowheads). B: A CoSA axon, in a $20 \mathrm{~h}$ embryo, has

contacted the central canal. The latter feature distinguishes them definitively from VeLD neurons. KA cells showed abnormalities both in axonal trajectory and in cell number.

In wildtype spinal cords $97 \%$ ( 65 of 67 ) of the KA neurons extended axons that ascended in the ipsilateral VLF. In the cyc- 1 spinal cord 17\% (6 of 36) of all $\mathrm{KA}$ axons were ipsilaterally descending rather than ipsilaterally ascending (Fig. 8D). The observation that some KA axons in the $c y c-1$ mutant were abnormal while many were normal is qualitatively similar to that seen for GABAergic CoSA neurons (above) and for VeLD and CoPA neurons (Bernhardt et al., '92).

Not only did some KA cells show axonal abnormalities, but there also was an approximately $40 \%$ reduction in their number as compared to the wildtype spinal cord (Table 2). KA neurons are the only class of spinal cells so far identified other than the midline floor plate cells that decrease in number in the $c y c-1$ spinal cord.

\section{DISCUSSION \\ Comparison of the cellular organization of the zebrafish and Xenopus spinal cords}

Two of the four classes of GABA-labeled spinal neurons in the zebrafish, DoLA and KA, have obvious homologues in Xenopus, both by the criteria of cellular morphology and immunoreactivity. DoLA in the zebrafish and a neuron in the dorsal cord of Xenopus with a similar morphology are both GABA immunoreactive (Roberts et al., '87). In Xenopus these dorsal, ascending neurons may also have a high affinity uptake system for GABA (Lamborghini and Iles, '85). KA cells in Xenopus (Dale et al., '87a,b; Roberts et al.,

failed to cross the ventral midline, instead it ascends ipsilateral to the parent cell body. More rostrally another CoSA growth cone ascends contralaterally, after having looped at the ventral midline. C: A VeLD neuron (asterisk) has an abnormal, ascending axon in this $22 \mathrm{~h}$ spinal cord. Normal DoLA (arrows) and KA neurons (arrowheads) are also indicated. D: An abnormal KA neuron (arrowhead) in a $22 \mathrm{~h}$ spinal cord has a descending axon which emerges laterally from the cell body. Another KA neuron, located more caudally, is normal. Magnification bar, $50 \mu \mathrm{m}$.

'87) and ventral medial cells identified as KA cells in zebrafish are both GABA immunoreactive. KA cells in Xenopus also have a high affinity GABA uptake mechanism (Lamborghini and Iles, '85). Although there are no reports of GABA immunoreactive commissural spinal cells and of possible VeLD homologues in Xenopus, some Xenopus commissural and unidentified ventral neurons do have a high affinity GABA uptake system (Lamborghini and Iles, '85). These neurons may represent counterparts to GABAergic CoSA and VeLD neurons of the zebrafish. The similarities in the distribution patterns of GABAergic neurons in the fish and amphibian spinal cords and our observation that the GABA antibody recognizes the same classes of neurons both in the wildtype and cyc-1 mutant zebrafish emphasize the usefulness of this marker in identifying specific neurons even when their cellular environment has been altered, either by evolution or by a mutation.

\section{The neuronal organization of the spinal cord is affected by the cyc-1 mutation}

The cyc-1 mutation in zebrafish leads to a floor plate deficit in the spinal cord. The floor of the wildtype cord of early zebrafish embryos usually consists of a row of neuroepithelial cells, approximately three cells wide in cross section, that run along the anterior/posterior axis and are bounded by longitudinal tracts (Kuwada et al., '90). The middle cell is morphologically and antigenically distinct from other spinal cells (Hatta et al., '91; Bernhardt et al., '92). The floor plate has been defined as consisting only of the midline cell (Hatta et al., '91) or as consisting of all three cells in the ventral floor (Kuwada et al., '90). The present results indicate that the lateral floor plate cells do not form an 
uninterrupted row, but that KA neurons are occasionally interspersed between them. The $c y c-1$ mutation deletes the midline floor plate cell throughout the rostrocaudal extent of the spinal cord (Hatta et al., '91). While it has been unclear whether cells immediately lateral to the midline floor plate cell are also affected, an examination of the morphologies of the cell types and locations of major axonal tracts suggested that the overall cellular organization of the mutant cord was normal (Bernhardt et al., '92; Eisen, '91; Hatta et al., '91).

Our findings on the numbers of GABAreactive cells in the wildtype and $c y c-1$ spinal cord generally corroborate the earlier findings. However, there is one major exception. Although the number of GABA immunoreactive DoLA, CoSA, and VeLD neurons was not affected, there was a substantial reduction in the number of KA neurons. This could be due to a direct effect of the mutation or be the indirect result of the deletion of the midline floor plate cells. In the latter case the midline floor plate cell would be required to induce the normal complement of KA cells. This is intriguing in light of the fact that the floor plate induces motor neurons in the chick spinal cord (Yamada et al., '91) and likely does the same in Xenopus (Clarke et al., '91) and the mouse (Bovalenta and Dodd, '91).

The fact that the distribution of several classes of dorsal neurons was not affected in $c y c-1$ spinal cords was expected since for the most part dorsal neurons are thought to develop normally even in absence of the notochord and of the floor plate in the chick (Yamada et al., '91; but see Hirano et al., '91), and mouse (Bovalenta and Dodd, '91). However, it has been somewhat of a puzzle that the number of primary motor neurons in cyc-1 spinal cords is apparently normal (Eisen, '91; Bernhardt et al., '92) given the observations in the chick, mouse, and Xenopus. It has been suggested that since the primary motor neurons are born so early in neurogenesis in the zebrafish, they may develop constitutively in the neuroectoderm without a requirement for either the notochord or the floor plate (Lumsden, '91). In fact, in Xenopus embryos the loss of the notochord and floor plate due to UV-irradiation results primarily in a reduction of the smaller, later born secondary motor neurons (Clarke et al., ' 91 ). In the cyc-1 mutant zebrafish some secondary motor neurons are apparently present (Kimmel et al., '91) but whether full complement of secondary motor neurons is formed in the absence of a floor plate is at present unknown. One interpretation of the finding of a reduced number of KA cells in $c y c-1$ embryos is that the floor plate may play a role in inducing the differentiation of relatively late developing ventral neurons. That the floor plate contributes to the survival of KA cells, seems less likely because there is no prominent cell death in the early cyc-1 spinal cord (Hatta et al., '91). A direct effect of the cyc-1 mutation also seems less probable because only a minority of the KA neurons is affected.

\section{Abnormal axonal outgrowth in the cyc-1 mutant spinal cord}

The axons of the GABAreactive CoSA and KA neurons followed either appropriate pathways or aberrant ones in $c y c-1$ embryos. The pathfinding errors of the GABAergic axons fell into three categories. 1) Some CoSA axons failed to extend directly to the ventral midline but instead extended longitudinally, either anteriorly or posteriorly, from the cell bodies before turning towards the ventral midline. 2) Some CoSA axons made laterality errors, i.e., they failed to cross the ventral midline. 3) Some CoSA and some KA axons made polarity errors near the ventral midline; i.e., they turned posteriorly rather than anteriorly. This is similar to the growth cones of the CoPA and VeLD neurons in cyc-1 embryos, two of the earliest neurons to initiate axonogenesis in the spinal cord (Bernhardt et al., '92). As with GABAreactive axons, CoPA and VeLD axons made errors both in laterality and polarity at the ventral midline. CoPA and VeLD axons could also be induced to follow the aberrant pathways by laser ablation of the midline floor plate cells in wildtype embryos (Bernhardt et al., '92). Together with control ablations, this demonstrated that aberrant axon trajectories can be due to the removal of the midline floor plate cells. These findings are corroborated by the abnormal axonal trajectories seen in the hindbrain of zebrafish embryos in the absence of the floor plate (Hatta et al., '90) and by a report of abnormal KA axonal outgrowth in Xenopus embryos missing both the floorplate and the notochord following early UV-irradiation (Clarke et al., '91).

The percentage and range of abnormalities of the CoPA and GABAreactive CoSA neurons seen in cyc-1 embryos are comparable: $58 \%$ of CoSA axons followed aberrant pathways, while $57 \%$ of CoPA axons did and both made errors in laterality and/or occasionally in polarity. Some differences between the abnormalities reported for the two cell types were, however, apparent. The most frequent aberrant CoPA trajectory was a contralateral, ascending one that remained in the ventral cord for an abnormally long distance, before extending into the dorsal cord. Because GABA-labeled CoSA neurons were scored at earlier stages of axonogenesis and the contralateral portions of the axons were still short, it was not possible to determine the extent to which axons remained in the ventral spinal cord. Therefore, we did not distinguish between normal or aberrant ventral axons and the proportion of aberrant CoSA axons may actually be higher. CoSA axons also followed trajectories in $c y c-1$ embryos that were not observed with CoPA axons. CoSA axons sometime extended longitudinally from the cell body before turning ventrally. CoPA axons, on the other hand, always extended directly toward the ventral midline.

Based on the analysis of axonal outgrowth by the CoPA and VeLD neurons it was suggested that the midline floor plate cells provide contact-mediated axonal guidance cues which lead to cell specific pathway choices at the ventral midline (Bernhardt et al., '92). Furthermore, it was hypothesized that additional guidance cues must be present at or near the ventral midline since a considerable number of growth cones followed normal pathways even in the absence of the midline floor plate cells. The finding of both normal and abnormal CoSA and KA axons, and the observation that most abnormalities in CoSA and KA pathfinding occurred at or near the ventral midline, support both aspects of this model.

A second way in which the floor plate can guide growing axons is by a long-range signal. In the mammalian and chick spinal cords, a chemotropic factor derived from the floor plate attracts the growth cones of commissural neurons to the ventral midline. In absence of the long-range attractant commissural axons often show abnormal trajectories in the ventral spinal cord (Tessier-Lavigne et al., ' 88 ; Placzek et al., '90; Bovalenta and Dodd, '91). The finding that some zebrafish CoSA axons reach the ventral midline by following abnormal, indirect routes could be explained if 
such an attractant was missing or diminished in the cyc-1 spinal cord. Thus, the available data suggests that the midline floor plate cells in the zebrafish spinal cord play both a long range and a contact-mediated role in the guidance of specific axons.

\section{ACKNOWLEDGMENTS}

We thank J. Kanki and R. Hume for helpful discussions. The work was supported by grants from the National Institutes of Health (NS 24848). CPK was supported by a grant from the National Science Foundation (DIR-9014275) to the research training group in Developmental Neurobiology at the University of Michigan.

\section{LITERATURE CITED}

Bernhardt, R.R., A B Chitnis, L Lindamer, and J.Y Kuwada (1990) Identification of spinal neurons in the embryonic and larval zebrafish. J. Comp. Neurol. 302:603-616.

Bernhardt, R.R., N. Nguyen, and J.Y. Kuwada (1992) Growth cone guidance by floor plate cells in the spinal cord of zebrafish embryos. Neuron 8:869-882.

Bovalenta, P., and J. Dodd (1990) Guidance of commissural growth cones at the floor plate in embryonic rat spinal cord. Development 109:435-447.

Bovalenta, P., and J. Dodd (1991) Perturbation of neuronal differentiation and axon guidance in the spinal cord of mouse embryos lacking a floor plate: Analysis of Danforth's short-tail mutation. Development 113:625639 .

Chitnis, A.B., and J.Y. Kuwada (1990) Axonogenesis in the brain of zebrafish embryos. J. Neurosci. 10:1892-1905.

Chitnis, A.B., and J.Y. Kuwada (1991) Elimination of a brain tract increases errors in axonal pathfinding by follower growth cones in the zebrafish embryo. Neuron 7:277-285.

Clarke, J.D.W., N. Holder, S.R. Soffe, and J. Storm-Mathisen (1991) Neuroanatomical and functional analysis of neural tube formation in notochordless Xenopus embryos; laterality of the ventral spinal cord is lost. Development 112:499-516

Dale, N., A. Roberts, O.P. Ottersen, and J. Storm-Mathisen (1987a) The morphology and distribution of 'Kolmer-Agduhr cells', a class of cerebrospinal-fuid-contacting neurons revealed in the frog embryo spinal cord by GABA immunohistochemistry. Proc. Royal Soc. Lond. B 232:193203.

Dale, N., A. Roberts, O.P. Ottersen, and J. Storm-Mathisen (1987b) The development of a population of spinal cord neurons and their axonal projections revealed by GABA immunocytochemistry in frog embryos Proc. Royal Soc. Lond. B 232:205-215.

Eisen, J.S. (1991) Developmental neurobiology of the zebrafish. J. Neurosci. 11:311-317.

Gallagher, B.C., and S. Moody (1987) Development of substance P-like immunoreactivity in Xenopus embryos. J. Comp. Neurol. 260:175-185.

Goodman, C.S., M. Bastiani, C.Q. Doe, S. du Lac, S. Helfand, J.Y. Kuwada and J.B. Thomas (1984) Cell recognition during neuronal development. Science 225:1271-1279.
Hatta, K., R.K. Ho, C. Walker, and C. Kimmel (1990) A mutation that deletes the floor plate and disturbs axonal pathfinding in zebrafish. Soc. Neurosci. Abstr. 16:310.

Hatta, K., C.B. Kimmel, R.K. Ho, and C. Walker (1991) The cyclops mutation blocks specification of the floor plate of the zebrafish central nervous system. Nature 350:3390-341.

Hirano, S., S. Fuse, G.S. Sohal (1991) The effect of the floor plate on pattern and polarity in the developing central nervous system. Science 251:310313.

Kimmel, C.B., K. Hatta, and J. Eisen (1991) Genetic control of primary neuronal development in zebrafish. Development Suppl. 2:47-57.

Kuwada, J.Y., R.R. Bernhardt, and A.B. Chitnis (1990) Pathfinding by identified growth cones in the spinal cord of zebrafish embryos. J. Neurosci. 10:1299-1308.

Lamborghini, J.E., and A. Iles (1985) Development of a high-affinity GABA uptake system in embryonic amphibian spinal neurons. Develop. Biol. 112:167-176

Lumsden, A. (1991) Motorizing the spinal cord. Cell 64:471-473.

Myers, P.Z., J.S. Eisen, and M. Westerfield (1986) Development and axonal outgrowth of identified motoneurons in zebrafish. J. Neurosei. $6: 2278-$ 2289 .

Patel, N.H., E. Martin-Blanco, K.G. Coleman, S.J. Poole, M.C. Ellis, T. B. Kornberg, and C.S. Goodman (1989) Expression of engrailed protein in arthropods, annelids, and chordates. Cell 58:955-968.

Piperno, G., and M.T. Fuller (1985) Monoclonal antibodies specific for an acetylated form of alpha-tubulin recognize the antigen in cilia and flagella from a variety of organisms. J. Cell Biol. 101:2085-2094.

Placzek, M., M. Tessier-Lavigne, T. Jessell, and J. Dodd (1990) Orientation of commissural axons in vitro in response to a floor plate-derived chemoattractant. Development 110:19-30.

Roberts, A., N. Dale, O.P. Ottersen, and J. Storm-Mathisen (1987) The early development of neurons with GABA immunoreactivity in the CNS of Xenopus laevis embryos. J. Comp. Neurol. 261:435-449.

Roberts, A., N. Dale, O.P. Ottersen, and J. Storm-Mathisen (1988) Development and characterization of commissural interneurons in the spinal cord of Xenopus laevis embryos revealed by antibodies to glycine. Development 103:447-461.

Rosenblatt, D., S. Murphy, R.R. Bernhardt, and J. Kuwada (submitted) Monoclonal antibody Mab CON1 labels subsets of neurons in the developing zebrafish central nervous system and recognizes neurofilament proteins.

Tessier-Lavigne, M., M. Placzek, A.G.S. Lumsden, J. Dodd, and T. Jessell (1988) Chemotropic guidance of developing axons in the mammalian central nervous system. Nature 336:775-778.

Van Straaten, H.M.W., J.W.M. Hekking, J.P.W.M. Beursgens, E. TerwindtRouwenhorst, and J. Drukker (1989) Effect of the notochord on proliferation and differentiation in the neural tube of the chick embryo. Development 107:793-803.

Yaginuma, H., and R.W. Oppenheim (1991) An experimental analysis of in vivo guidance cues used by axons of spinal interneurons in the chick embryo: Evidence for chemotropism and related guidance mechanisms. J. Neurosci. 11:2598-3613

Yamada, T., M. Placzek, H. Tanaka, J. Dodd, and T.M. Jessell (1991) Control of cell pattern in the developing nervous system: Polarizing activity of the floor plate and notochord. Cell 64:635-647. 